Research Article

\title{
INVESTIGATION OF THE EFFECT OF SHEAR THICKENING FLUID AND FABRIC STRUCTURE ON INTER-YARN FRICTION PROPERTIES IN TWARON FABRICS
}

\begin{abstract}
Cenk YANEN ${ }^{1}$, Murat Yavuz SOLMAZ ${ }^{2}$, Ercan AYDOĞMUŞ ${ }^{3}$
It is known that shear thickening fluids increase the energy absorption capacity of high performance fabrics. For this reason, soft body armors have been produced by impregnating shear thickening fluids recently on high performance fabrics. In this study, it is aimed to examine the effects of shear thickening fluid and fabric structure on the inter-yarn friction properties of para-aramid fabrics with different properties. Fumed silica was dispersed in polyethylene glycol to produce shear thickening fluid. Twaron 200 and Twaron 460 para-aramid fabrics with different properties such as threads number (105x105 and 67x67), areal density (200 gr $/ \mathrm{m}^{2}$ and $\left.460 \mathrm{gr} / \mathrm{m}^{2}\right)$ and linear density (930 and 3360 Dtex) were impregnated with shear thickening fluid. It was observed that both of shear thickening fluid impregnated fabrics had higher yarn pull-out force than neat fabrics due to inter-yarn friction. When shear thickening fluid impregnated fabrics are according to maximum yarn pull-out force; Twaron 200 and Twaron 460 fabrics requires approximately 3 times and 9 times more force to pull-out the yarn in comparison to the neat fabric respectively. In this study, it has been observed that the positive effects of shear thickening fluid impregnation on energy absorption of Twaron fabrics depend on structural parameters such as threads number, areal density and linear density.
\end{abstract}

Key words: shear thickening fluid, pull-out test, inter-yarn friction, silica, polyethylene glycol

\section{Introduction}

Shear thickening fluids are a non-Newtonian fluid type obtained by homogeneous mixing of nanosized solid particles in a liquid medium. The viscosity of these fluids increases suddenly under increasing shear rate and when this shear rate disappears, their viscosity returns to its original state. Many researches have been done to explain these remarkable behaviour of non-Newtonian fluids. The first of these researches was carried out by Hoffman. In the study carried out by Hoffmann, researchers stated that the solid particles in the suspension were in an orderly manner under the point called critical shear rate. Hoffman reported that over this point, the particle arrangement was disrupted and irregular particle clusters were formed. This idea forms the basis of the Order - Disorder Theory [1-3]. In subsequent studies, it has been reported that strong hydrodynamic forces occur in the suspension under high stresses

\footnotetext{
${ }^{1}$ Department of Mechanical Engineering, Firat University, 23100 Elazig, Turkey, (cyanen@firat.edu.tr) (Dhttps://orcid.org/00000002-5092-8734

${ }^{2}$ Department of Mechanical Engineering, Firat University, 23100 Elazig, Turkey, (mysolmaz@ firat.edu.tr) (Dhttps://orcid.org/ 00000001-6394-0313

${ }^{3}$ Department of Chemical Engineering, Firat University, 23100 Elazig, Turkey, (ercanaydogmus@ firat.edu.tr) (Dhttps://orcid.org/ 0000-0002-1643-2487
} 
and therefore the particles come into contact with each other. As a result, it has been reported that structures formed as a result of the clustering of many particles, called hydrocluster, are formed in suspension. This theory is called the hydroclustering theory and it is generally accepted for explaining the structure of STFs [4-6].

Shear thickening fluids have been used in many areas due to their unique properties mentioned above. Personal body armors, protective clothings and energy absorbers are the areas where these fluids are most widely used. Many scientific researches have been conducted to examine the development of energy absorption capacity by impregnating STF on high performance fabrics such as Kevlar $[7,8]$, Twaron [9,10] and ultra high moleculer weight polyethylene (UHMWPE) [11,12] used in personal body armors. Majumdar et al. investigated the deformation and energy absorption capacity of STF impregnated and neat Kevlar woven fabrics after impact damage. It was found that in neat Kevlar fabrics only primary yarns participated in load sharing and hence energy absorption. However, it was reported that in STF impregnated fabrics, unlike neat fabrics, not only primary yarns but also entire fabric participates in load bearing and energy absorption [13]. Park et al. investigated the effects of neat and STF impregnated Kevlar fabrics on high speed impact energy absorption. STF impregnation was found to provide a significant increase in energy absorption. It was reported that thinner shielding configurations with equivalent energy absorption performance were possible through STF impregnation, and the STF impregnated Kevlar fabric provides approximately $70 \%$ improved specific energy absorption performance over neat Kevlar [14]. The effects of different silica particles and changes in solid particle ratios on rheological properties and energy absorption capacity in quasi-static puncture tests were studied. It has been reported that as the amount of silica in STF increases, the critical shear rate decreases and the viscosity increases. It has been reported that the quasi-static puncture resistance of STF impregnated Twaron fabrics is significantly increased [15-17]. Li et al. applied dynamic impact tests to STF impregnated UHMPWE fabrics. The effect of UHMWPE fabrics on energy absorption against knife and spike threats and the role of STF in this event were studied in detail. The results showed that the dynamic knife resistance of the UHMWPE fabric increased significantly due to the presence of imregnated STF. STF has been reported to effectively reduce yarn mobility and accelerate the transverse response of the UHMWPE fabric and increase the inter-yarn friction, thus making the impactor point pushing the yarns to the edge and making it difficult to penetrate the fabric [18].

The reasons for this energy absorption effect obtained by impregnating STF with high performance fabrics were investigated. Gürgen and Kuşhan produced single and multi-solid phase STFs in their study. They reported that STF impregnated Twaron fabrics were more successful in ballistic and dynamic impact tests than neat fabrics. It was reported in the study that there is no connection between energy absorption and rheological properties. When the yarn friction test results were examined, it was found that they were similar to both ballistic and dynamic impact test results. It was concluded that STFs increase friction by penetrating into the yarns, therefore inter-yarn friction is decisive in the energy absorption effect [19-20].

In the literature review, limited number of studies are found on the energy absorption effect of the physical properties of the fabrics. Zhang et.al investigated the effect of fabric structure and thickness on the ballistic impact behavior of UHMWPE composite laminate [21]. Arora et al. performed pull-out and drop tower tests on STF impregnated UHMWPE fabrics produced from al 400 denier and 1350 denier yarn. In the pull-out tests of fabrics produced with both yarns, success was observed in STFimpregnated fabrics compared to neat fabrics. However, in impact tests, it was reported that STF impregnation on fabrics produced with 400 denier reduces the energy absorption capacity [22]. 
According to most researches in the literature, it has been reported that STF has a positive effect on energy absorption. In addition to causing an increase in viscosity at high strain rates, the STF process also affects inter-yarn friction, which significantly affects the impact resistance performance of fabrics against both low and high speed attacks. The inter-yarn friction level can be used as an indicator to estimate the energy absorption performance of any fabric. In this study, yarn pull-out tests were carried out by impregnating STF on two different Twaron para-aramid fabrics. It has been investigated whether the positive effects of STFs on the energy absorption of Twaron fabrics are dependent on structural parameters such as threads number, areal density and linear density.

\section{Experimentals}

\subsection{Materials}

Fumed silica (Aerosil 150) which has a particle size of $14 \mathrm{~nm}$ and a specific surface area of 150 $\mathrm{m}^{2} / \mathrm{g}$ was used as solid particle in the production of STF. Polyethylene glycol (PEG) with average molecular weight $400 \mathrm{~g} / \mathrm{mol}$ was used as a liquid medium. Two different Twaron para-aramid fabrics produced by Teijin Limited were used in the study. Table 1 presents the manufacturer's specification for the Twaron 460 and Twaron 200. Schematic representation of Twaron fabrics is given in Figure 1.
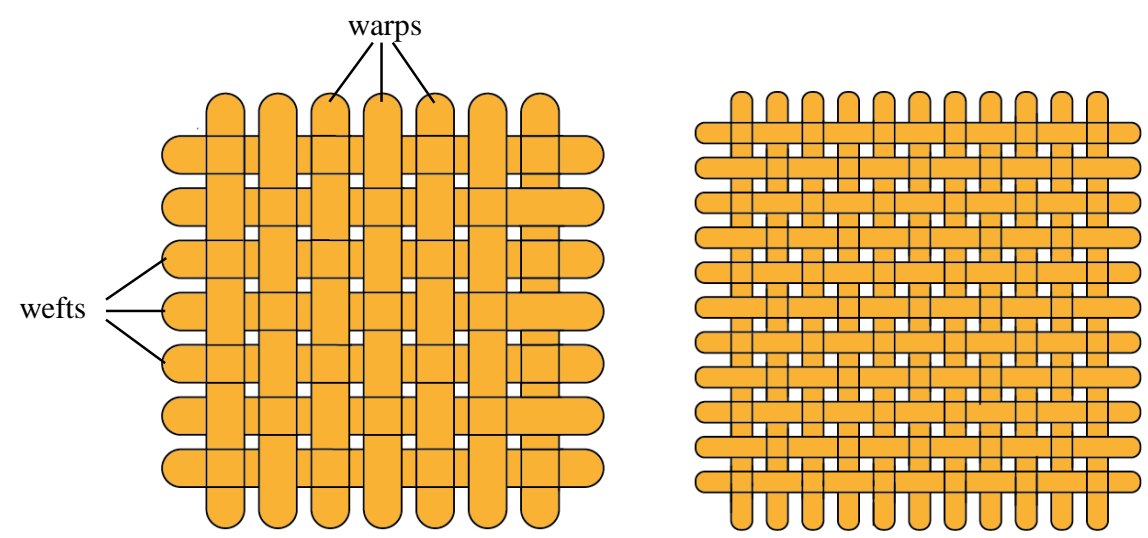

Figure 1. Schematic representation of fabrics a) Twaron 460 b) Twaron 200.

Table 1. Details of Twaron para-aramid fabrics

\begin{tabular}{ccc}
\hline Parameters & Twaron 460 & Twaron 200 \\
\hline Weave & Plain & Plain \\
Areal density $\left(\mathrm{g} / \mathrm{m}^{2}\right)$ & 460 & 200 \\
Linear density $(\mathrm{Dtex})$ & 3360 & 930 \\
Threads $($ per $10 \mathrm{~cm})$ & $67 \times 67$ & $105 \times 105$ \\
Thickness $(\mathrm{mm})$ & 0.4 & 0.2 \\
\hline
\end{tabular}

\subsection{Preparation of STF}

Silica particles, were dried in oven at $150^{\circ} \mathrm{C}$ for about 12 hours to get rid of moisture adsorbed on the surface. Polyethylene glycol and silica particles were mixed by using a high speed mechanical mixer to prepare the STF samples. The mixing speed was kept constant at $6000 \mathrm{rpm}$. During the mixing process, silica particles were gradually added to prevent agglomeration as suggested in previous studies $[19,23,24]$ The mixing process was continued until the mixture became homogeneous. The amount of fumed silica particles in the suspension was $25 \mathrm{wt} \%$. 


\subsection{Preparation of STF-Treated Fabric}

In order to impregnate Twaron para-aramid fabric with STF, the prepared STFs were diluted in ethanol at a 1:1 approximate weight ratio of STF:ethanol. A high-speed mechanical mixer (6000 rpm) was used to avoid the agglomeration of silica particles and to ensure uniform dispersion. The Twaron para-aramid fabric was cut into rectangle specimens of $20 \mathrm{~mm} \times 200 \mathrm{~mm}$. Each of the fabrics were immersed into the diluted STF for $1 \mathrm{~min}$. Finally, the fabrics were dried at $79{ }^{\circ} \mathrm{C}$ for 2 hours in an oven to evaporate the ethanol from the fabrics.

\subsection{Rheological analysis of STF}

Rheological properties of STF were determined using Anton Paar MCR 102 tension controlled rheometer. Tests were carried out using a $25 \mathrm{~mm}$ diameter parallel plate apparatus. The gap between the plates was kept constant at $0.3 \mathrm{~mm}$ and all tests were conducted at $25^{\circ} \mathrm{C}$. Rheological measurements were made in the range of $0-1000 \mathrm{~s}^{-1}$ shear rate.

\subsection{Pull-out test}

Pull-out tests were carried out using Shimadzu tensile testing machine with a $10 \mathrm{kN}$ capacity to study the effect of inter-yarn friction. The specimens were cut in 20x200 mm dimensions. A single yarn thread was tied to the upper jaw of tensile test machine and pulled through the fabric while keeping the lower jaw fixed as suggested in previous studies [25-28]. In the experiments, the preload value was used as $0.1 \mathrm{~N}$ and the crosshead speed as $100 \mathrm{~mm} / \mathrm{min}$. Pull-out tests were carried out on both STF impregnated and neat fabric. Fig. 2 shows yarn pull-out test and the schematic representation of the specimens used in the pull-out test.

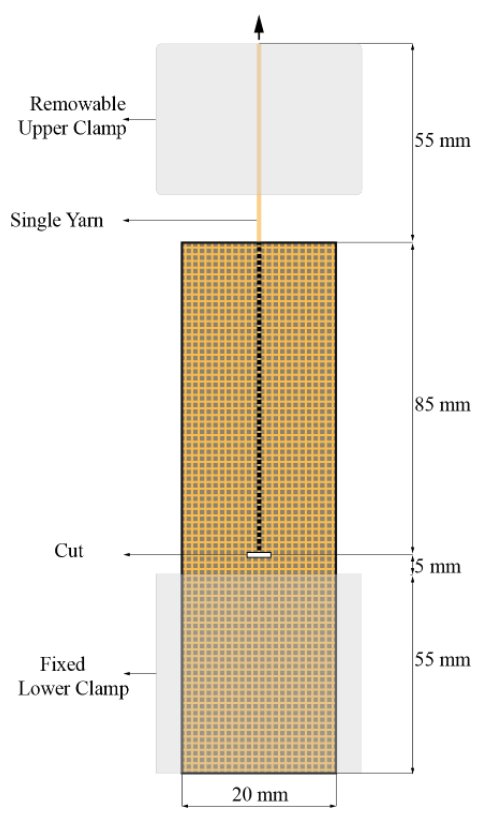

(a)

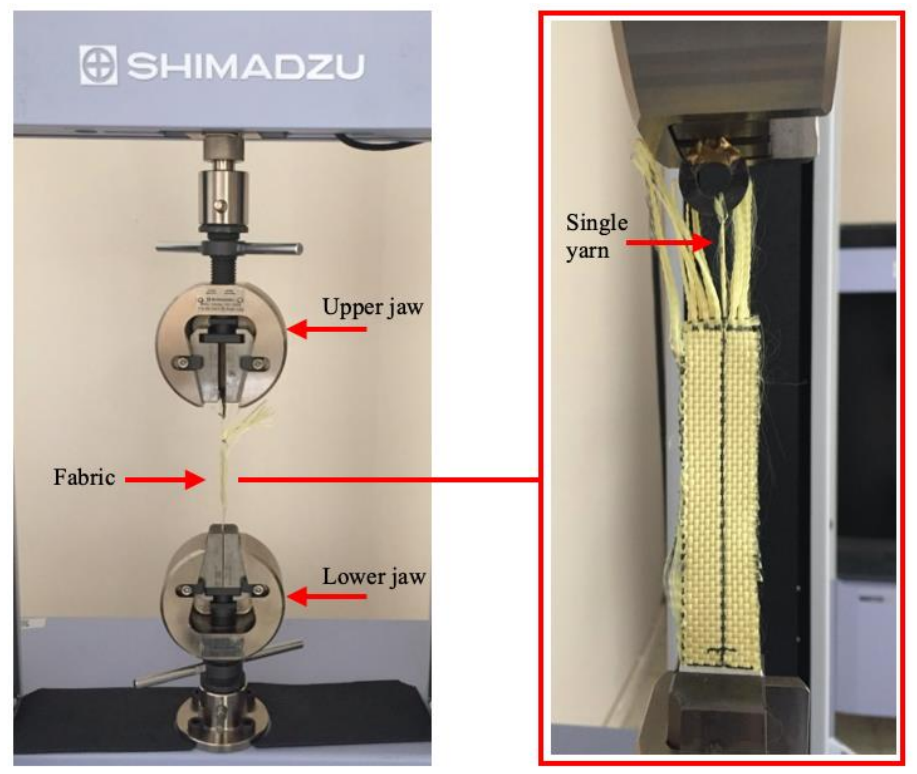

(b)

Figure 2. (a) schematic representation of the specimen (b) yarn pull-out test. 


\section{Results and discussion}

\subsection{Rheological analysis}

When the rheological test results shown in Fig. 3 are examined, it can be said that PEG shows Newtonian behavior, and when fumed silica particles are included to PEG, the suspension shows nonNewtonian behavior. The initial viscosity of STF is 135 Pa.s and showed shear thinning behavior under up to $51.5 \mathrm{~s}^{-1}$ shear rate. After this critical shear rate, there is a sudden increase in viscosity and the peak viscosity is measured 178 Pa.s. After reaching peak viscosity, shear thinning behavior occurred in the suspension, and it is determined that the final viscosity is 112 Pa.s.

The hydro-cluster theory is based on the interactions of solid particles in a liquid medium. With the increased shear rate, the particles suspended in the fluid contact each other and cause an increase in hydrodynamic forces. The ratio between hydrodynamic forces and Brownian forces determines the properties of the solidification mechanism. This ratio of hydrodynamic forces to Brownian forces gives information about the rheology of the suspension. At low shear rates, the suspension microstructure begins to deteriorate and Brownian forces of the silica particles increase. This causes a decrease in suspension viscosity, that is shear thinning. However, as the shear rate increases, the hydrodynamic forces appear more dominant in the suspension. When the point called critical shear rate is reached, the hydrodynamic forces increase to a level that allows the silica particles to aggregate. Thus, the Brownian forces of the silica particles are reduced and as a result, the hydro clusters formed in the suspension cause the viscosity to increase as barriers that prevent the liquid flow.

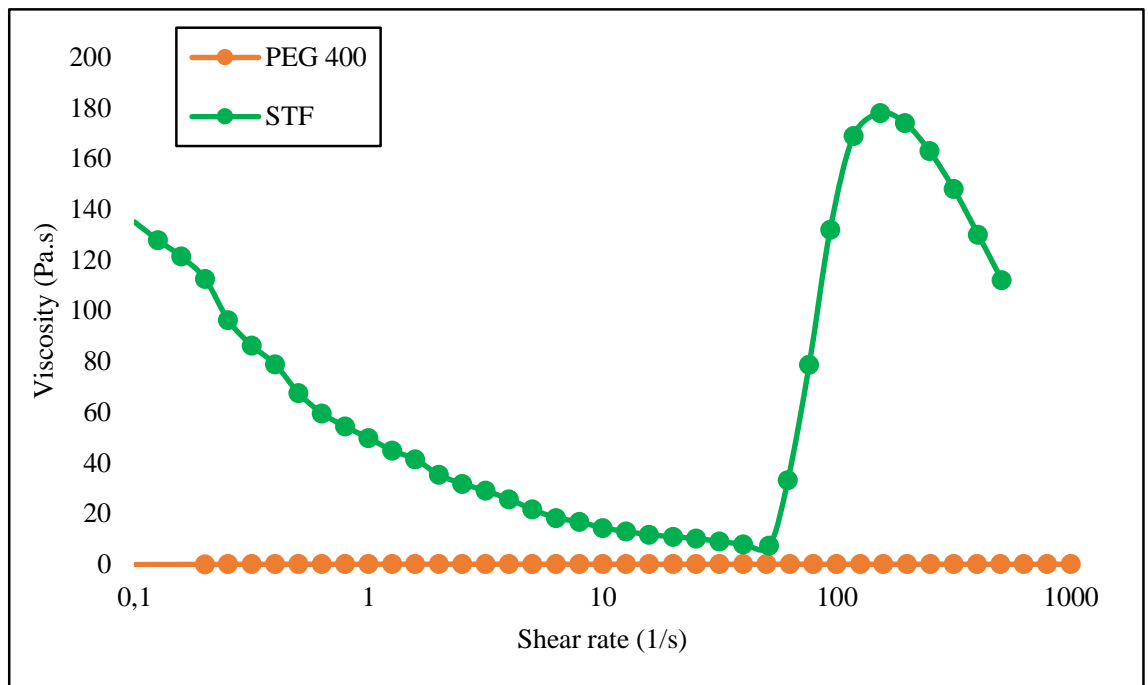

Figure 3. Rheological curves of STF and PEG.

\subsection{Scanning electron microscopy (SEM)}

The SEM images were presented in Fig. 4. Surface morphology of neat and STF impregnated Twaron fabrics was evaluated by using Zeiss Evo MA10 scanning electron microscope (SEM). The samples were plated with gold to make them conductive. The samples were properly dried at $79{ }^{\circ} \mathrm{C}$ for 12 hours in an oven to evaporate the ethanol from the fabrics before testing. Fig. 4a and Fig. 4b show neat Twaron para-aramid fabrics without any treatment. Fig. 4c and Fig. 4d show that STF penetrates between the fibers in the fabric in the SEM images of STF imregnated Twaron fabrics. 


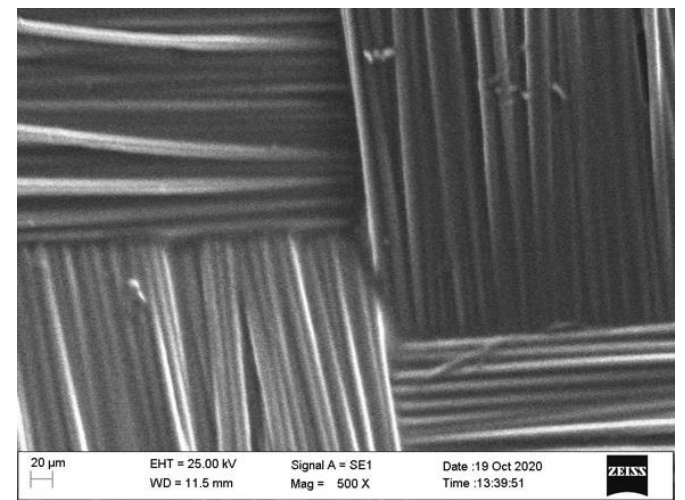

(a)

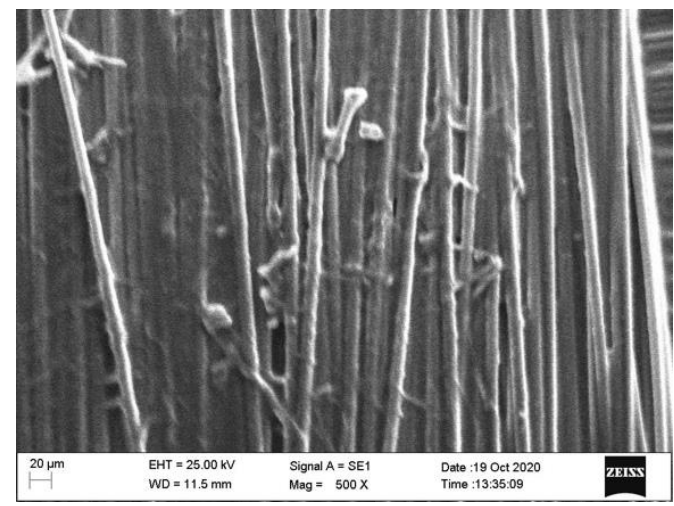

(c)

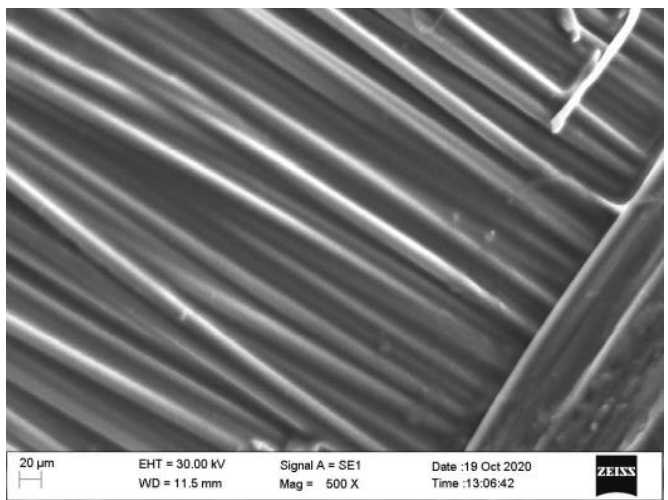

(b)

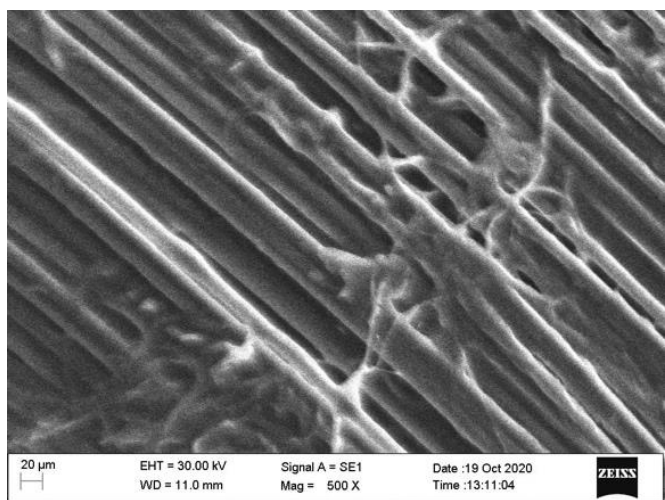

(d)

Figure 4. SEM image of the (a) Twaron 200 neat fabric (b) Twaron 460 neat fabric (c) Twaron 200 STF impregnated fabric (d) Twaron 460 STF impregnated fabric.

\subsection{Effect of fabric structure on pull-out force}

As stated in previous researches, the yarn pull-out test results are as much important as the mechanical properties of the high-performance fabrics in the knife and spike puncture and ballistic resistance experiments. Some previous investigations, it is stated that the major contribution in explaining the role of STFs in energy absorption is not due to shear thickening behavior, but to the increase in inter-yarn friction $[19,26]$. Therefore yarn pull-out tests were performed to evaluate the inter-yarn friction of the neat and STF impregnated Twaron para-aramid fabrics.

The behaviors of fabrics in the yarn pull-out tests are given in Fig.5. At the beginning of the testing, since the number of crossing yarns that a single yarn will pass through is maximum, the pullout force increases until it reaches the peak point. When the pull-out force reaches its peak point, the first crossing yarn is passed and the single yarn starts to oscillating. The pull-out force decreases gradually. With each crossing yarn the force continues to decrease and oscillating motion is seen. This movement continues until the single yarn is completely removed from the fabric weave.

When the pull-out test results are examined, it can be said that fabrics with STF impregnated were more successful than neat fabrics for both fabrics. Fig. 6 shows that maximum pull-out force of Twaron 200 and Twaron 460 neat fabrics are measured as $1.64 \mathrm{~N}$ and $2.12 \mathrm{~N}$, respectively. Twaron 460 neat fabric requires $29 \%$ higher yarn pull-out force in comparison to the Twaron 200 neat fabric. Maximum pull-out force of Twaron 200 and Twaron 460 fabrics impregnated with shear thickening fluid are measured as $6.79 \mathrm{~N}$ and $20.96 \mathrm{~N}$, respectively. STF impregnated fabrics as is the case with neat fabrics, 
Twaron 460 fabric performs better than Twaron 200 fabric in pull-out tests. According to the maximum pull-out force; STF impregnated Twaron 200 and Twaron 460 fabrics show increase 314\% and 889\% compared to neat fabrics, respectively.

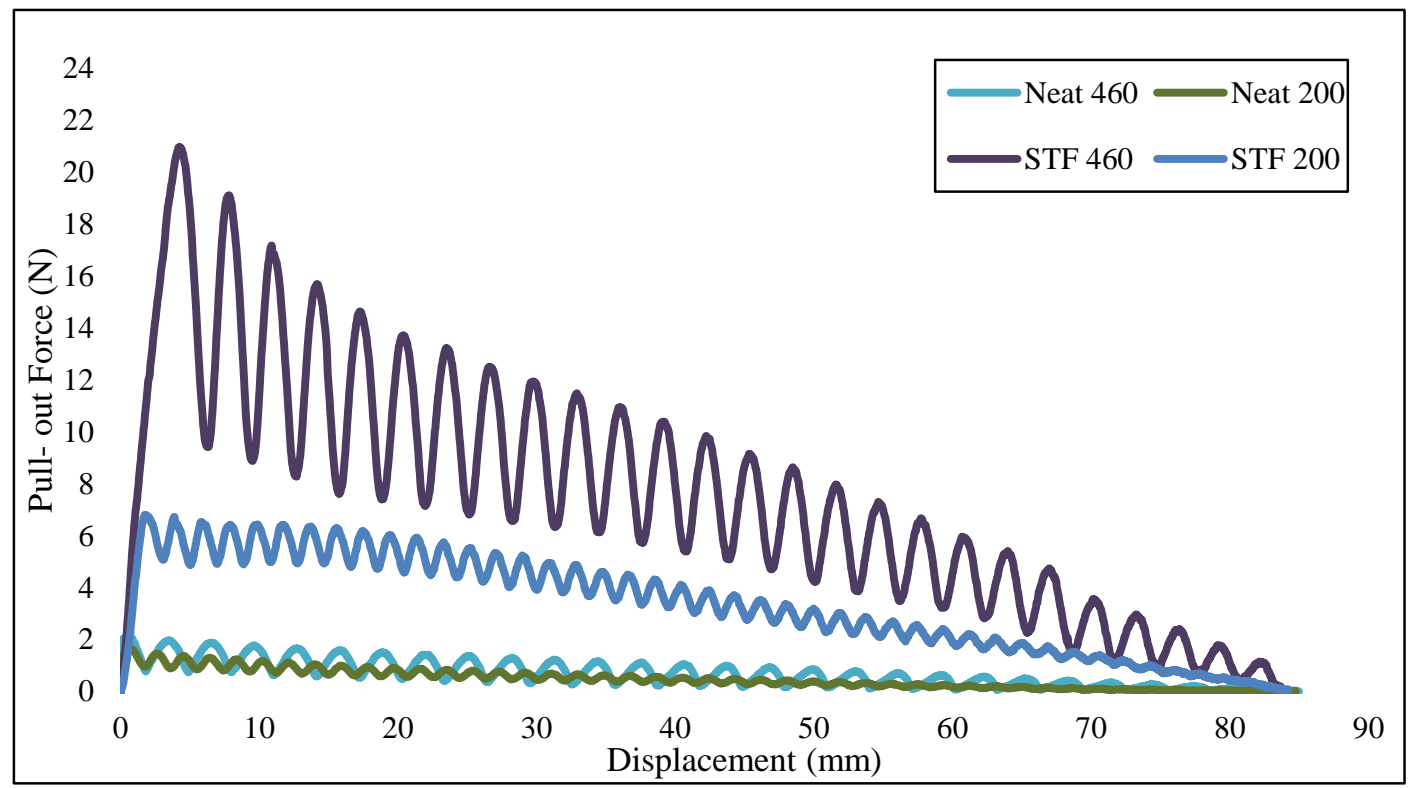

Figure 5. Force - displacement behaviour during yarn pull-out test results for each fabrics.

Because 930 Dtex yarns are thinner than 3360 Dtex yarns, the contact area between yarns is much lower as compared to that of 3360 Dtex yarn. For this reason, Twaron 200 has a lower yarn pull-out force than Twaron 460. When the number of weft and warp in the fabrics is low, the transition points of the single yarn between warp and weft yarns are also less. The yarn pull-out force is related to the number of the transition points between the weft and warp yarns. Therefore, less force is required to pull a yarn from fabric with a lower number of weft and warp. When STF is impregnated on Twaron 460 fabric with a lower number of weft and warp $(67 \times 67$ per $10 \mathrm{~cm})$, the inter-yarn friction increases significantly as it will be relatively easy to penetration between the yarns. Consequently, the increase in yarn pull-out force is quite high. When STF is impregnated on Twaron 200 fabric with a higher number of weft and warp (105x105 per $10 \mathrm{~cm})$, the inter-yarn friction also increases. However, since the number of transition points is high, it is more difficult for STF to penetrate between the yarns. For this reason, impregnation of STF can increase the friction effect between yarns to a more limited extent.

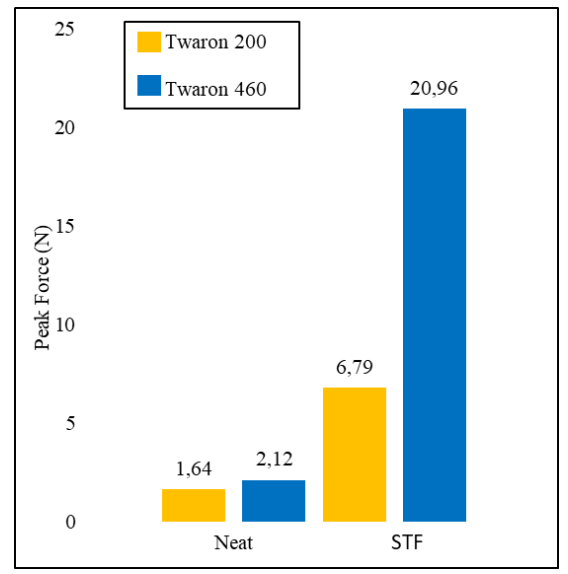

Figure 6. Yarn pull-out force. 


\section{Conclusions}

Fumed silica was dispersed in polyethylene glycol to produce shear thickening fluid. Twaron 200 and Twaron 460 para-aramid fabrics with different properties such as threads (105x105, 67x67), areal density $\left(200 \mathrm{~g} / \mathrm{m}^{2}, 460 \mathrm{~g} / \mathrm{m}^{2}\right)$ and linear density (930, 3360 Dtex) were impregnated with STF. Both of STF impregnated fabrics have higher yarn pull-out force than neat fabrics, which were due to the increase for inter-yarn friction. Twaron 460 fabric impregnated with STF increased the maximum yarn pull-out force at a higher rate than the Twaron 200 fabric compared to the neat fabric. In this study, it was seen that the positive effects of STFs on the energy absorption of Twaron fabrics depend on structural parameters such as threads, areal density and linear density. Rheological properties of multiphase STFs will be examined in future studies. Impact and ballistic tests of multi-phase STF impregnated fabrics will be performed and their efficiency in energy absorption will be investigated.

\section{Acknowledgment}

The authors gratefully acknowledge the financial support by the Research Fund of Firat University, Project FÜBAP-MF.19.44. The author Cenk Yanen also acknowledges the support of the Scientific and Technological Research Council of Turkey (TÜBITTAK) under Program 2211/C.

\section{References}

[1] R. L. Hoffman, Discontinuous and dilatant viscosity behavior in concentrated suspensions--1. Observation of a flow instability, Transactions of the Society of Rheology, 16 (1972), 1, https://doi.org/10.1122/1.549250

[2] R. L. Hoffman, Discontinuous and dilatant viscosity behavior in concentrated suspensions. II. Theory and experimental tests, J. Colloid Interface Sci., 46 (1974), 3, pp. 491-506, doi: 10.1016/0021-9797(74)90059-9.

[3] R. L. Hoffman, Explanations for the cause of shear thickening in concentrated colloidal suspensions, Journal of Rheology, 42 (1998), 1, doi: 10.1122/1.550884.

[4] G. Bossis and J. F. Brady, The rheology of Brownian suspensions, J. Chem. Phys., 91 (1989), 3, doi: 10.1063/1.457091.

[5] W. H. Boersma, J. Laven, and H. N. Stein, Viscoelastic properties of concentrated shearthickening dispersions, J. Colloid Interface Sci., 149 (1992), 1, pp. 10-22, doi: 10.1016/00219797(92)90385-Y.

[6] J. R. Melrose, J. H. van Vliet, and R. C. Ball, Continuous shear thickening and colloid surfaces, Phys. Rev. Lett., 77 (1996), 22, doi: 10.1103/PhysRevLett.77.4660.

[7] R. Bai, Y. Ma, Z. Lei, Y. Feng, and C. Liu, Energy analysis of fabric impregnated by shear thickening fluid in yarn pullout test, Compos. Part B Eng., 174 (2019), doi: 10.1016/j.compositesb.2019.106901.

[8] U. Mawkhlieng and A. Majumdar, Deconstructing the role of shear thickening fluid in enhancing the impact resistance of high-performance fabrics, Compos. Part B Eng., 175 (2019), doi: 10.1016/j.compositesb.2019.107167. 
[9] H. R. Baharvandi, M. Alebooyeh, M. Alizadeh, M. S. Heydari, N. Kordani, and P. Khaksari, The influences of particle-particle interaction and viscosity of carrier fluid on characteristics of silica and calcium carbonate suspensions-coated Twaron ${ }^{\circledR}$ composite, J. Exp. Nanosci., 11 (2015), 7, pp. 550-563, doi: 10.1080/17458080.2015.1094190.

[10] S. Gürgen and M. C. Kuşhan, The effect of silicon carbide additives on the stab resistance of shear thickening fluid treated fabrics, Mech. Adv. Mater. Struct., 24 (2017), 16, pp. 1381-1390, doi: 10.1080/15376494.2016.1231355.

[11] L. L. Sun, D. S. Xiong, and C. Y. Xu, Application of shear thickening fluid in ultra high molecular weight polyethylene fabric, J. Appl. Polym. Sci., 129 (2013), 4, pp. 1922-1928, doi: 10.1002/app.38844.

[12] N. Asija, H. Chouhan, S. Amare Gebremeskel, and N. Bhatnagar, Impact response of Shear Thickening Fluid (STF) treated ultra high molecular weight poly ethylene composites - study of the effect of STF treatment method, Thin-Walled Struct., 126 (2018), pp. 16-25, doi: 10.1016/j.tws.2017.04.025.

[13] Majumdar, B. S. Butola, and A. Srivastava, An analysis of deformation and energy absorption modes of shear thickening fluid treated Kevlar fabrics as soft body armour materials, Mater. Des., 51 (2013), pp. 148-153, doi: 10.1016/j.matdes.2013.04.016.

[14] Y. Park, Y. Kim, A. H. Baluch, and C. G. Kim, Empirical study of the high velocity impact energy absorption characteristics of shear thickening fluid (STF) impregnated Kevlar fabric, Int. J. Impact Eng., 72 (2014), pp. 67-74, doi: 10.1016/j.ijimpeng.2014.05.007.

[15] H. R. Baharvandi, P. Khaksari, M. Alebouyeh, M. Alizadeh, J. Khojasteh, and N. Kordani, Investigating the quasi-static puncture resistance of $\mathrm{p}$-aramid nanocomposite impregnated with the shear thickening fluid, J. Reinf. Plast. Compos., 33 (2014), 22, pp. 2064-2072, doi: 10.1177/0731684414554635.

[16] H. R. Baharvandi, M. Alebooyeh, M. Alizadeh, P. Khaksari, and N. Kordani, Effect of silica weight fraction on rheological and quasi-static puncture characteristics of shear thickening fluidtreated Twaron ${ }^{\circledR}$ composite, J. Ind. Text., 46, (2016), 2, pp. 473-494, doi: $10.1177 / 1528083715589750$.

[17] H. R. Baharvandi, M. Saeedi Heydari, N. Kordani, M. Alebooyeh, M. Alizadeh, and P. Khaksari, Characterization of the rheological and mechanical properties of shear thickening fluid-coated Twaron ${ }^{\circledR}$ composite, J. Text. Inst., 108, (2017) 3, pp. 397-407, doi: 10.1080/00405000.2016.1168091.

[18] W. Li, D. Xiong, X. Zhao, L. Sun, and J. Liu, Dynamic stab resistance of ultra-high molecular weight polyethylene fabric impregnated with shear thickening fluid, Mater. Des., 102 (2016), pp. 162-167, doi: 10.1016/j.matdes.2016.04.006.

[19] S. Gürgen and M. C. Kuşhan, The stab resistance of fabrics impregnated with shear thickening fluids including various particle size of additives, Compos. Part A Appl. Sci. Manuf., 94 (2017), pp. 50-60, doi: 10.1016/j.compositesa.2016.12.019. 
[20] S. Gürgen and M. C. Kuşhan, The ballistic performance of aramid based fabrics impregnated with multi-phase shear thickening fluids, Polym. Test., 64 (2017), pp. 296-306, doi: 10.1016/j.polymertesting.2017.11.003.

[21] D. Zhang, Y. Sun, L. Chen, S. Zhang, and N. Pan, Influence of fabric structure and thickness on the ballistic impact behavior of Ultrahigh molecular weight polyethylene composite laminate, Mater. Des., 54 (2014), pp. 315-322, doi: 10.1016/j.matdes.2013.08.074.

[22] S. Arora, A. Majumdar, and B. S. Butola, Structure induced effectiveness of shear thickening fluid for modulating impact resistance of UHMWPE fabrics, Compos. Struct., 210 (2019), pp. 41-48, doi: 10.1016/j.compstruct.2018.11.028.

[23] X. Z. Zhang, W. H. Li, and X. L. Gong, The rheology of shear thickening fluid (STF) and the dynamic performance of anSTF-filled damper, Smart Mater. Struct., 17 (2008), 3, doi: 10.1088/0964-1726/17/3/035027.

[24] Y. Xu, Stabbing Resistance of Soft Ballistic Body Armour Impregnated with Shear Thickening Fluid, Ph. D. thesis, University of Manchester, Manchester, UK, 2016

[25] Y. Sun, R. Wang, X. Liu, M. Li, H. Yang, and B. Li, Improvements in the thermal conductivity and mechanical properties of phase-change microcapsules with oxygen-plasma-modified multiwalled carbon nanotubes, J. Appl. Polym. Sci., 134 (2017), 44, doi: 10.1002/app.45269.

[26] Q. shi Wang, R. jun Sun, M. Yao, M. yu Chen, and Y. Feng, The influence of temperature on inter-yarns fictional properties of shear thickening fluids treated Kevlar fabrics, Compos. Part A Appl. Sci. Manuf., 116 (2019), pp. 46-53, doi: 10.1016/j.compositesa.2018.10.020.

[27] M. Fahool and A. R. Sabet, Parametric study of energy absorption mechanism in Twaron fabric impregnated with a shear thickening fluid, Int. J. Impact Eng., 90 (2016), pp. 61-71, doi: 10.1016/j.ijimpeng.2015.11.016.

[28] X. Gong, Y. Xu, W. Zhu, S. Xuan, W. Jiang, and W. Jiang, Study of the knife stab and punctureresistant performance for shear thickening fluid enhanced fabric, J. Compos. Mater., 48 (2014), 6, pp. 641-657, doi: 10.1177/0021998313476525. 